\section{Análise temporal da desigualdade em escolaridade no tabagismo e consumo abusivo de álcool nas capitais brasileiras}

\author{
Time trend in inequalities in smoking and abusive \\ alcohol consumption in Brazil's state capitals
}

\section{Tendencia temporal de las desigualdades en el tabaquismo y consumo abusivo de alcohol en capitales brasileñas}

\author{
Andrea Wendt 1 \\ Caroline S. Costa 2 \\ Francine S. Costa 1 \\ Deborah Carvalho Malta ${ }^{3}$ \\ Inácio Crochemore-Silva 1
}

doi: 10.1590/0102-311X00050120

\section{Resumo}

Estudo de tendência temporal com o objetivo de avaliar as desigualdades no tabagismo e no consumo abusivo de álcool, considerando a escolaridade como proxy de nível socioeconômico, de acordo com sexo e regiões brasileiras. Foram utilizados dados do Vigilância de Fatores de Risco e Proteção para Doenças Crônicas por Inquérito Telefônico (Vigitel) coletados entre 2006 e 2017. Os desfechos foram o consumo abusivo de bebidas alcoólicas e o fumo. As desigualdades foram avaliadas com base na escolaridade, com dupla estratificação por sexo e regiões brasileiras. Para avaliar a tendência das prevalências dos desfechos e suas desigualdades foi usada a regressão linear ponderada pelos quadrados mínimos da variância. A prevalência de consumo abusivo de álcool aumentou em mulheres, de 7,8\% (2006) para 12,2\% (2017), e foi maior sempre entre os mais escolarizados. A prevalência de tabagismo diminuiu para homens e mulheres e foi maior sempre entre os menos escolarizados. A desigualdade no consumo de álcool entre os grupos de escolaridade parece ter aumentado ao longo do tempo, com maior magnitude entre as mulheres, mas a desigualdade no tabagismo parece estar diminuindo. Em algumas regiões do país, as medidas de desigualdade sugerem estabilidade e em outras um aumento da desigualdade para o consumo de álcool (Sudeste, Sul e Centro-oeste, entre as mulheres) e diminuição para o tabagismo (todas as regiões, exceto Sudeste, entre os homens; Nordeste e Centro-oeste, entre as mulheres). Apesar dos avanços na redução do tabagismo, as desigualdades na escolaridade persistem e estão presentes também em relação ao álcool. Os desfechos têm comportamentos opostos, portanto, estratégias devem ser focadas em cada um dos problemas, a fim de reduzir as desigualdades existentes.

Tabagismo; Fatores de Risco; Doença Crônica; Indicadores de Desigualdade em Saúde

\author{
Correspondência \\ A. Wendt \\ Programa de Pós-graduação em Epidemiologia, Universidade \\ Federal de Pelotas. \\ Rua Marechal Deodoro 1160, 3o andar, Pelotas, RS 96020-220, \\ Brasil. \\ andreatwendt@gmail.com \\ 1 Programa de Pós-graduação em Epidemiologia, Universidade \\ Federal de Pelotas, Pelotas, Brasil. \\ 2 Faculdade de Saúde Pública, Universidade de São Paulo, Rio de \\ Janeiro, Brasil. \\ 3 Programa de Pós-graduação em Saúde Pública, Universidade \\ Federal de Minas Gerais, Belo Horizonte, Brasil.
}




\section{Introdução}

O impacto que as doenças crônicas não transmissíveis (DCNT) têm na qualidade de vida e nas taxas de mortalidade é uma preocupação crescente, principalmente em vista do atual contexto político e econômico baseado em medidas de austeridade no Brasil. Ainda que tenha havido uma redução na mortalidade atribuída às DCNT no Brasil, estima-se que estas sejam responsáveis por cerca de $72 \%$ das mortes 1. Projeções para o ano de 2030 foram realizadas, considerando cenários hipotéticos de transferência federal de fundos para municípios e para quatro indicadores de saúde, incluindo mortalidade evitável por doenças cardiovasculares. Os resultados mostram uma deterioração nos indicadores de saúde, mais fortemente em municípios menores, exacerbando as desigualdades geográficas 2. As regiões Norte e Nordeste, por exemplo, apresentaram os piores indicadores em relação às demais, segundo inquéritos domiciliares realizados de 1998 a 2013 3 2

No mundo, $78 \%$ das mortes por DCNTs ocorreram em países de baixa e média renda ${ }^{4}$. A morbimortalidade por DCNT é maior em populações socioeconomicamente mais vulneráveis 5 e as iniquidades relacionadas à mortalidade poderiam ser explicadas, em parte, pela ocorrência de fatores de risco modificáveis 6 , como o fumo e o consumo abusivo de álcool. Além de serem drogas lícitas fortemente associadas às DCNTs, álcool e fumo representam fatores de risco comuns para outros comportamentos de risco à saúde. Por isso, políticas de intervenção para a redução do consumo de álcool poderiam seguir estratégias semelhantes àquelas utilizadas para a diminuição do tabaco 7 .

Estudos prévios mostraram que o Brasil vivenciou ao longo dos anos um declínio na prevalência de tabagismo 8 , já a prevalência de consumo abusivo de álcool permaneceu constante ao longo dos anos ${ }^{9}$. No entanto, as prevalências desses fatores de risco para DCNT podem ser potencialmente diferentes em subgrupos populacionais, como os distintos estratos de escolaridade, inclusive dentro de cada região geográfica. Além disso, observa-se que tanto para o consumo de álcool quanto para o tabagismo existem diferenças importantes entre homens e mulheres 10. Assim, o monitoramento de estimativas globais em âmbito nacional pode esconder importantes desigualdades, as quais são passíveis de mudança ao longo do tempo 11.

Em vista da necessidade de elaboração de políticas e estratégias de intervenção, que visem ao declínio da morbimortalidade associada às DCNTs em função da redução de seus fatores de risco, e frente à situação atual do Brasil, é extremamente importante o monitoramento de fatores de risco e da evolução da magnitude das diferenças entre estratos de nível socioeconômico. Assim, o objetivo deste estudo é avaliar as desigualdades no tabagismo e no consumo abusivo de álcool, considerando a escolaridade como proxy de nível socioeconômico, de acordo com sexo e regiões brasileiras.

\section{Métodos}

O trabalho foi realizado com dados do Vigitel (Vigilância de Fatores de Risco e Proteção para Doenças Crônicas por Inquérito Telefônico), coletados entre 2006 e 2017, sobre a população adulta ( $\geq 18$ anos de idade) das 26 capitais dos estados brasileiros e do Distrito Federal. O Ministério da Saúde promove a coleta desses dados desde 2006, a fim de monitorar fatores de risco para doenças crônicas não transmissíveis, com base em uma amostra probabilística e representativa da população de adultos que residem em domicílios com cadastro de linha telefônica fixa. Em todos os anos, foi realizado sorteio sistemático e estratificado por CEP (código de endereçamento postal) das linhas telefônicas por cidade e sorteio de um dos adultos residentes no domicílio sorteado e elegível 12.

Os questionários, feitos por telefone, incluíam perguntas sobre características demográficas e socioeconômicas, uma série de comportamentos relacionados à saúde, bem como diagnóstico médico de doenças crônicas não transmissíveis autorreferido pelo entrevistado. Para o presente estudo foram usadas informações sociodemográficas e de consumo de cigarros e bebidas alcoólicas ${ }^{12}$. Pesos pós-estratificação foram calculados para cada indivíduo entrevistado, com o objetivo de ajustar a distribuição sociodemográfica da amostra Vigitel à distribuição da população adulta da cidade projetada 12,13.

O consentimento livre e esclarecido foi obtido oralmente no momento do contato telefônico com os entrevistados. O projeto Vigitel foi aprovado pela Comissão Nacional de Ética em Pesquisa para Seres Humanos do Ministério da Saúde (CAAE: 65610017.1.0000.0008). 
Para o presente trabalho, considerou-se como desfecho o consumo abusivo de bebidas alcoólicas, avaliado pela pergunta: "Nos últimos 30 dias, o sr. chegou a consumir cinco ou mais doses de bebida alcoólica em uma única ocasião?", para homens, ou: "Nos últimos 30 dias, a sra. chegou a consumir quatro ou mais doses de bebida alcoólica em uma única ocasião?”, para mulheres. Esse indicador é conhecido como consumo de álcool em binge, ou ingerir grandes quantidades em uma única ocasião, sendo muito frequente entre os jovens. O fumo atual, avaliado pela pergunta: "O(A) sr(a). fuma?", independentemente do número de cigarros, da frequência e da duração do hábito de fumar.

A tendência de desigualdades no período de 2006 a 2017 foi avaliada com base na escolaridade (0-8, 9-11, 12 ou mais anos completos de estudos), como proxy de nível econômico, sendo realizada dupla estratificação por sexo (masculino e feminino) e as capitais das regiões brasileiras (Norte, Nordeste, Sudeste, Sul e Centro-oeste). Para avaliar tanto a tendência das prevalências dos desfechos quanto das desigualdades, foi utilizada a regressão de Prais-Winsten que leva em conta a autocorrelação serial 14 considerando significativos os valores de $p<0,05$. As variáveis dependentes foram as prevalências e medidas de desigualdade, e a independente os anos dos inquéritos. Todos os valores $\mathrm{p}$ apresentados nas análises foram obtidos da regressão de Prais-Winsten, levando-se em conta todos os anos de inquérito (2006-2017).

A amostra foi descrita de acordo com a prevalência de consumo abusivo de álcool e de tabagismo total, e segundo escolaridade e sexo de 2006 a 2017. As medidas de desigualdades usadas foram o índice angular de desigualdade (IAD) e o índice relativo de desigualdade (IRD). Ambas são medidas sumárias de desigualdades para variáveis categóricas ordinais. O IAD estima, com base em modelos de regressão, a desigualdade absoluta em pontos percentuais (p.p.), variando de -100 a +100 , com resultados positivos indicando uma maior frequência do desfecho na população mais escolarizada e negativos na menos escolarizada. O IAD pode ser interpretado, por exemplo, como o montante em p.p. que a prevalência nos menos escolarizados foi maior do que nos mais escolarizados. O IRD é uma razão que identifica a desigualdade relativa, variando de 0 a $+\infty$, com resultados maiores que 1 indicando uma maior frequência do desfecho na população mais escolarizada, e menores que 1, uma maior frequência na população menos escolarizada. No IAD e no IRD, os valores 0 e 1 significam igualdade, respectivamente. IAD e IRD são medidas robustas baseadas em modelos de regressão, levando-se em consideração todas as categorias da variável e não apenas as extremas. Ainda, permitem a análise de variáveis desbalanceadas em termos de tamanho de amostra em cada categoria, como a escolaridade e, especialmente, considerando que uma mobilidade entre grupos de escolaridade é, geralmente, observada ao longo do tempo 15,16,17.

Todas as análises foram realizadas no software Stata versão 15.0 (https://www.stata.com), utilizando-se o comando survey devido ao processo de amostragem complexa.

\section{Resultados}

Desde 2006, o Vigitel inclui em sua amostra aproximadamente 50 mil indivíduos (menor tamanho de amostra em $2014=40.853$; maior em 2006 = 54.369). Em 2006, as proporções da amostra com menor escolaridade, escolaridade intermediária e maior escolaridade foram 45,5\%, 33,3\% e 21,2\%. Em 2017, esses três grupos foram correspondentes a $30,8 \%, 37,3 \%$ e $31,9 \%$.

A Figura 1 apresenta a prevalência total de consumo abusivo de álcool de acordo com os grupos de escolaridade. A prevalência total de consumo abusivo de álcool em homens variou de $25 \%$ em 2006 a 27,1\% em 2017, mas não apresentou mudança significativa ao longo dos anos ( $\mathrm{p}=0,735)$. Para as mulheres, a prevalência total de consumo abusivo de álcool manteve um padrão crescente, sendo 7,8\% em 2006 e 12,2\% em 2017 ( p < 0,001). Para os dois sexos, a prevalência no grupo mais escolarizado foi maior em todos os anos. Os valores do IAD e IRD para o consumo abusivo de álcool foram todos positivos e maiores que 1 , respectivamente, indicando maior frequência do desfecho entre os mais escolarizados (Tabela 1). Para o consumo abusivo de álcool em homens, o IAD foi 8,9 em 2006 e 12,7 em 2017. Nos anos de 2009, 2010 e 2016 foram identificados os maiores valores no IAD durante todo o período, com os mais escolarizados apresentando prevalência de consumo abusivo de álcool cerca de 16p.p. maiores, quando comparados aos menos escolarizados, mas com estabilidade deste padrão ao longo do tempo $(\mathrm{p}=0.177)$. A desigualdade relativa, verificada por meio do IRD, variou de $1,41 \mathrm{em}$ 
Figura 1

Consumo abusivo de álcool total, de acordo com escolaridade em homens e mulheres. Vigilância de Fatores de Risco e Proteção para Doenças Crônicas por Inquérito Telefônico (Vigitel), Brasil, 2006 a 2017.

1a) Homens

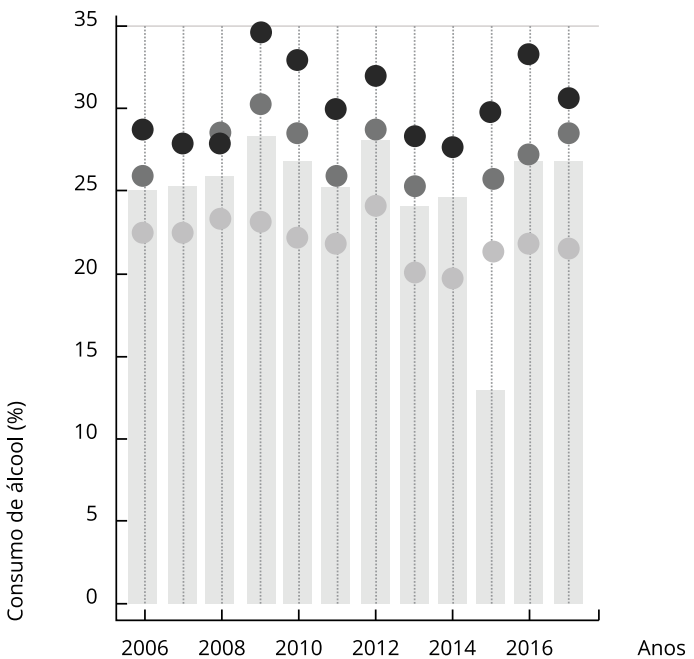

1b) Mulheres

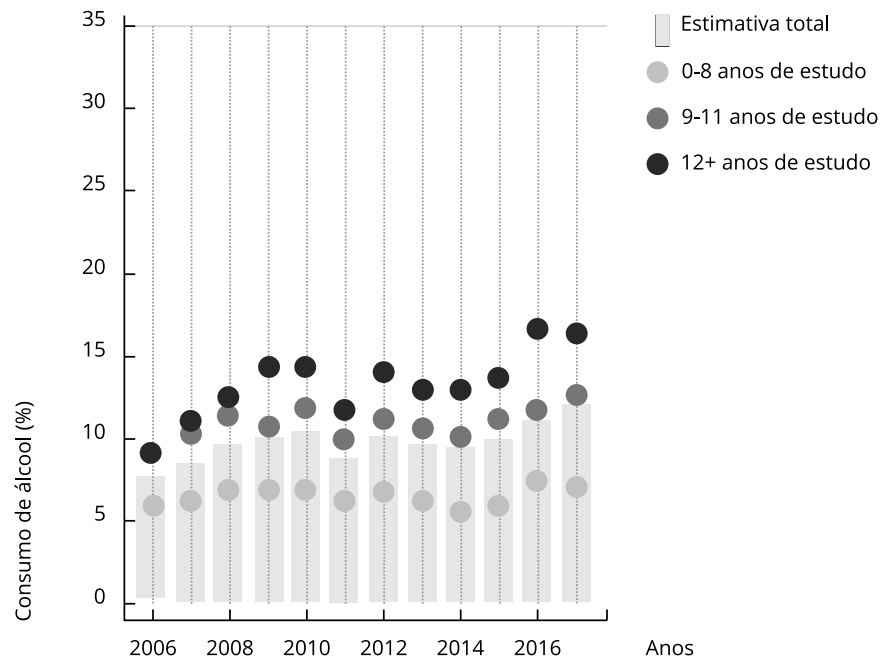

2006 a 1,58 em 2017, com maiores valores nos anos de 2009, 2010 e 2016, mas sem aumento significativo em todo o período ( $\mathrm{p}=0.583$ ). Para as mulheres, o IAD aumentou de 5,3 em 2006 para 14,2 em 2017, ou seja, a diferença na prevalência de consumo abusivo de álcool entre as mulheres mais e menos escolarizadas foi quase três vezes maior em 2017 ( $\mathrm{p}<0,001$ ). Já o IRD variou de 1,85 em 2006 a 2,93 em 2017, com aumento significativo ( $\mathrm{p}=0,021)$ (Tabela 1$)$.

A prevalência total de tabagismo de 2006 a 2017 e de acordo com os grupos de escolaridade é apresentada na Figura 2. Para os homens, o tabagismo diminuiu de 19,5\% em 2006 para 13,2\% em 2017 ( $\mathrm{p}<0,001$ ). Nas mulheres a mudança foi de 12,4\% em 2006 para 7,5\% em 2017 (p < 0,001). Para ambos os sexos, a prevalência foi maior sempre entre os menos escolarizados, com os grupos intermediário e mais escolarizado apresentando prevalências semelhantes (exceto para mulheres nos anos inicial e final). Para o tabagismo, as medidas de desigualdade mostraram padrão inverso ao consumo abusivo de álcool, sendo o tabagismo mais frequente entre os menos escolarizados (Tabela 1). Para os homens, o IAD variou de -17,6 em 2006 a -12,1 em 2017 (p < 0,001) e o IRD não apresentou mudança significativa, com valores de 0,41 em 2006 e 2017 ( $\mathrm{p}=0,583)$. Para as mulheres, o IAD variou de $-9,1$ em 2006 a -4,7 em 2017, sem mudanças significativas ( $\mathrm{p}=0,787$ ), já o IRD passou de 0,50 em 2006 para 0,56 em 2017 ( $\mathrm{p}<0,001)$.

A Tabela 2 apresenta a desigualdade absoluta, expressa pelo IAD, no consumo abusivo de álcool ao longo do período para cada região brasileira. Para os homens, as medidas apresentam grande flutuação ao longo do tempo em todas as regiões, assim como no país. Para todos os anos, o maior consumo de álcool ocorreu entre os mais escolarizados e apenas na Região Nordeste houve uma diminuição significativa na desigualdade ( $\mathrm{p}=0,005)$, embora a flutuação ainda esteja presente (IAD em $2006=$ 12,8p.p. maiores entre os mais escolarizados; IAD em $2017=12,1$ p.p. maiores entre os mais escolarizados). Para as mulheres, o maior consumo de álcool também foi maior entre as mais escolarizadas e a desigualdade aumentou nas regiões Sudeste ( $\mathrm{p}=$ 0,006; IAD em $2006=6$,1p.p. maiores entre as mais escolarizadas; IAD em 2017 = 14,9p.p. maiores entre as mais escolarizadas), Sul ( $<<0,001$; IAD em $2006=8$,4p.p. maiores entre as mais escolarizadas; IAD em $2017=17,4$ p.p. maiores entre as mais 


\section{Tabela 1}

Índice angular de desigualdade (IAD) e índice relativo de desigualdade (IRD) para consumo abusivo de álcool e tabagismo de acordo sexo. Vigilância de Fatores de Risco e Proteção para Doenças Crônicas por Inquérito Telefônico (Vigitel), Brasil, 2006 a 2017.

\begin{tabular}{|c|c|c|c|c|}
\hline & \multicolumn{2}{|c|}{ Homens } & \multicolumn{2}{|c|}{ Mulheres } \\
\hline & IAD (IC95\%) & IRD (IC95\%) & IAD (IC95\%) & IRD (IC95\%) \\
\hline \multicolumn{5}{|c|}{ Consumo abusivo de álcool } \\
\hline 2006 & $8,9(4,6 ; 13,2)$ & $1,41(1,17 ; 1,66)$ & $5,3(3,3 ; 7,3)$ & $1,85(1,41 ; 2,29)$ \\
\hline 2007 & $9,0(4,9 ; 13,1)$ & $1,42(1,19 ; 1,64)$ & $7,5(5,3 ; 9,6)$ & $2,20(1,68 ; 2,71)$ \\
\hline 2008 & $8,0(3,8 ; 12,2)$ & $1,35(1,13 ; 1,57)$ & $8,6(6,3 ; 10,9)$ & $2,28(1,77 ; 2,79)$ \\
\hline 2009 & $16,9(12,4 ; 21,5)$ & $1,81(1,51 ; 2,12)$ & $10,5(8,1 ; 12,8)$ & $2,63(2,05 ; 3,23)$ \\
\hline 2010 & $15,4(10,9 ; 19,9)$ & $1,76(1,46 ; 2,07)$ & $11,0(8,6 ; 13,4)$ & $2,68(2,10 ; 3,26)$ \\
\hline 2011 & $11,3(7,0 ; 15,7)$ & $1,56(1,29 ; 1,83)$ & $8,5(6,3 ; 10,7)$ & $2,39(1,86 ; 2,92)$ \\
\hline 2012 & $11,0(5,8 ; 16,2)$ & $1,47(1,20 ; 1,75)$ & $10,5(7,7 ; 13,2)$ & $2,58(1,94 ; 3,22)$ \\
\hline 2013 & $11,7(7,4 ; 16,0)$ & $1,60(1,32 ; 1,89)$ & $9,9(7,3 ; 12,4)$ & $2,54(1,93 ; 3,16)$ \\
\hline 2014 & $12,3(7,2 ; 17,4)$ & $1,62(1,29 ; 1,95)$ & $10,5(7,5 ; 13,5)$ & $2,78(1,97 ; 3,60)$ \\
\hline 2015 & $11,7(6,6 ; 16,8)$ & $1,58(1,25 ; 1,90)$ & $11,3(8,6 ; 14,0)$ & $2,81(2,08 ; 3,54)$ \\
\hline 2016 & $16,4(11,6 ; 21,2)$ & $1,80(1,48 ; 2,11)$ & $13,6(10,4 ; 16,8)$ & $2,83(2,14 ; 3,50)$ \\
\hline \multirow[t]{2}{*}{2017} & $12,7(7,8 ; 17,6)$ & $1,58(1,30 ; 1,87)$ & $14,2(10,8 ; 17,6)$ & $2,93(2,19 ; 3,67)$ \\
\hline & $p=0,177$ & $p=0,583$ & $p<0,001$ & $p<0,001$ \\
\hline \multicolumn{5}{|c|}{ Tabagismo } \\
\hline 2006 & $-17,6(-21,9 ;-13,3)$ & $0,41(0,33 ; 0,50)$ & $-9,1(-11,9 ;-6,4)$ & $0,50(0,40 ; 0,60)$ \\
\hline 2007 & $-15,1(-19,3 ;-10,9)$ & $0,47(0,37 ; 0,56)$ & $-6,3(-9,1 ;-3,5)$ & $0,62(0,49 ; 0,74)$ \\
\hline 2008 & $-19,5(-23,8 ;-15,1)$ & $0,34(0,27 ; 0,42)$ & $-7,5(-10,3 ;-4,8)$ & $0,55(0,43 ; 0,67)$ \\
\hline 2009 & $-15,5(-20,0 ;-11,1)$ & $0,42(0,32 ; 0,52)$ & $-8,6(-11,5 ;-5,7)$ & $0,49(0,38 ; 0,61)$ \\
\hline 2010 & $-16,5(-21,0 ;-12,0)$ & $0,38(0,29 ; 0,48)$ & $-8,7(-11,6 ;-5,8)$ & $0,50(0,39 ; 0,61)$ \\
\hline 2011 & $-16,2(-20,4 ;-12,0)$ & $0,39(0,29 ; 0,48)$ & $-10,7(-13,7 ;-7,7)$ & $0,39(0,29 ; 0,49)$ \\
\hline 2012 & $-14,4(-19,3 ;-9,4)$ & $0,41(0,29 ; 0,53)$ & $-8,5(-11,3 ;-5,8)$ & $0,43(0,31 ; 0,54)$ \\
\hline 2013 & $-13,6(-17,6 ;-9,7)$ & $0,40(0,29 ; 0,50)$ & $-8,8(-11,4 ;-6,3)$ & $0,39(0,29 ; 0,49)$ \\
\hline 2014 & $-12,5(-16,5 ;-8,5)$ & $0,39(0,28 ; 0,51)$ & $-9,0(-12,1 ;-5,9)$ & $0,40(0,27 ; 0,52)$ \\
\hline 2015 & $-12,2(-16,5 ;-7,8)$ & $0,41(0,29 ; 0,53)$ & $-9,2(-12,3 ;-6,2)$ & $0,36(0,25 ; 0,47)$ \\
\hline 2016 & $-11,7(-15,6 ;-7,9)$ & $0,40(0,29 ; 0,52)$ & $-9,3(-11,9 ;-6,7)$ & $0,34(0,24 ; 0,44)$ \\
\hline \multirow[t]{2}{*}{2017} & $-12,1(-16,1 ;-8,1)$ & $0,41(0,29 ; 0,53)$ & $-4,7(-7,2 ;-2,3)$ & $0,56(0,39 ; 0,74)$ \\
\hline & $p<0,001$ & $p=0,583$ & $p=0,787$ & $p<0,001$ \\
\hline
\end{tabular}

Nota: valores positivos (IAD) ou maiores que 1 (IRD) indicam maior frequência do desfecho entre os mais escolarizados e valores negativos (IAD) ou menores que 1 (IRD), nos menos escolarizados; os valores de $p$ são referentes a tendência linear do IAD e IRD ao longo do tempo, obtidos através da regressão de Prais-Wisten.

escolarizadas) e Centro-oeste ( $\mathrm{p}=0,001$; IAD em 2006 = 3,1p.p. maiores entre as mais escolarizadas; IAD em $2017=17,1$ p.p. maiores entre as mais escolarizadas).

As desigualdades absolutas para tabagismo nas regiões brasileiras são apresentadas na Tabela 2. Tanto para homens quanto para mulheres a prevalência de tabagismo foi maior entre os menos escolarizados para todos os anos, o que é evidenciado nos IAD negativos. Para os homens, houve diminuição na desigualdade para as regiões Norte ( $\mathrm{p}=$ 0,004, IAD em 2006 = 23,3p.p. maiores entre os menos escolarizados; IAD em 2017 = 16,5p.p. maiores entre os menos escolarizados), Nordeste ( $\mathrm{p}=$ 0,015; IAD em 2006 = 13,7p.p. maiores entre os menos escolarizados; IAD em $2017=$ 13,1p.p. maiores entre os menos escolarizados), $\mathrm{Sul}(\mathrm{p}=0,001$; IAD em $2006=31$,5p.p. maiores entre os menos escolarizados; IAD em 2017 = 14,1p.p. maiores entre os menos escolarizados) e Centro-oeste $(\mathrm{p}<$ 0,001; IAD em 2006 = 22,8p.p. maiores entre os menos escolarizados; IAD em $2017=6$,8p.p. maiores entre os menos escolarizados). Para as mulheres, a diminuição da desigualdade ao longo dos 
Figura 2

Tabagismo total de acordo com escolaridade em homens e mulheres. Vigilância de Fatores de Risco e Proteção para Doenças Crônicas por Inquérito Telefônico (Vigitel), Brasil, 2006 a 2017.

2a) Homens

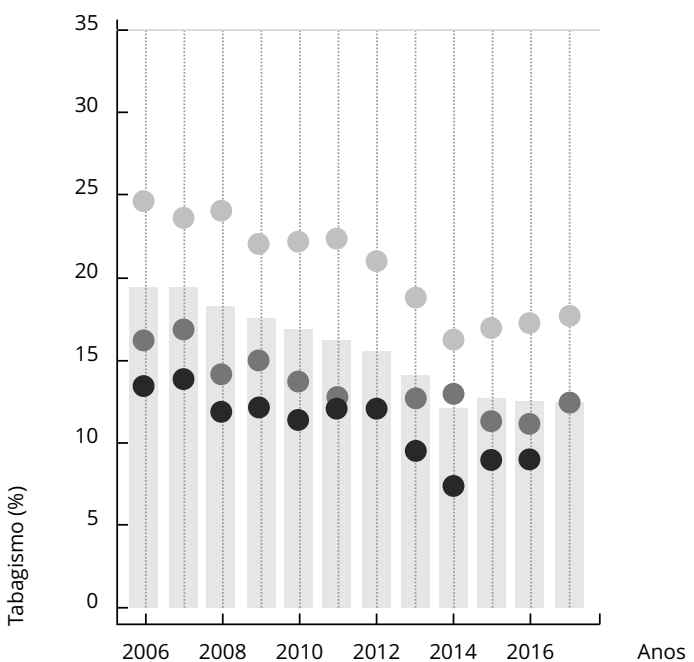

2b) Mulheres

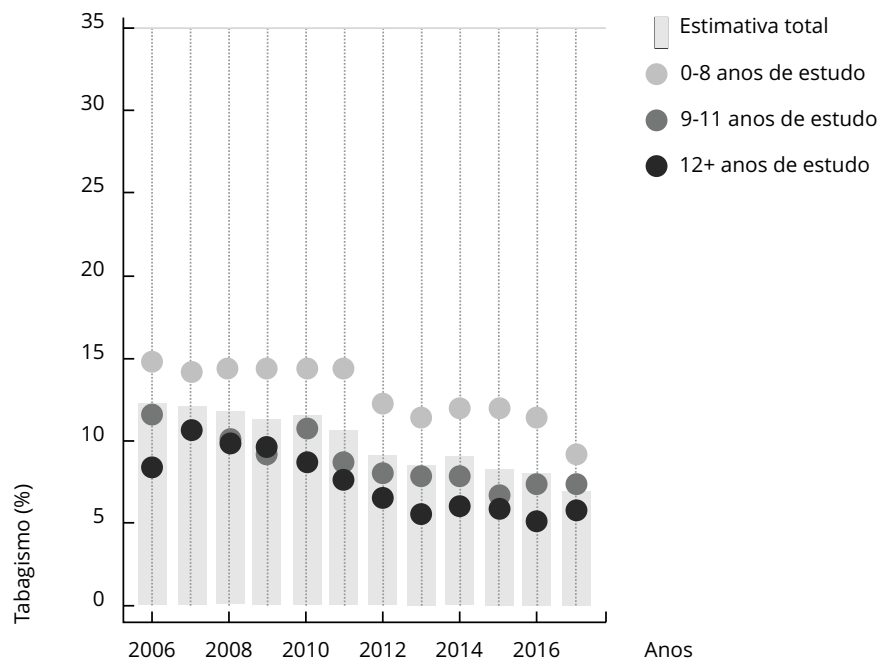

anos foi observada apenas nas regiões Nordeste ( $\mathrm{p}=0,002$; IAD em $2006=12,8 \mathrm{p} . \mathrm{p}$. maiores entre as menos escolarizadas; IAD em 2017 = 4,7p.p. maiores entre as menos escolarizadas) e Centro-oeste $(\mathrm{p}=0,013 ; \mathrm{IAD}$ em $2006=10,8$ p.p. maiores entre as menos escolarizadas; IAD em $2017=9,3$ p.p. maiores entre as menos escolarizadas).

\section{Discussão}

Os resultados do presente trabalho mostraram uma prevalência de consumo abusivo de álcool maior sempre entre os mais escolarizados, já a prevalência de tabagismo foi maior sempre entre os menos escolarizados. Independentemente da escolaridade, os homens apresentaram estimativas maiores que as mulheres nos dois comportamentos de saúde estudados. A desigualdade entre os grupos de escolaridade para o consumo abusivo de álcool parece ter aumentado ao longo do tempo, com maior magnitude de aumento entre as mulheres. Já a desigualdade no tabagismo parece estar diminuindo, assim como a prevalência total do desfecho. Em relação às regiões do país, um padrão claro de aumento ou diminuição das desigualdades não foi identificado, mas em algumas localidades as medidas sugerem estabilidade e em outras um padrão semelhante ao resto do país.

Em relação ao álcool, destaca-se que não existem níveis seguros de consumo e seus efeitos incluem aumento do risco para doenças infecciosas, doenças não transmissíveis e lesões 18,19 . Devido à sua importância como fator de risco, o álcool foi incluído entre as metas dos Objetivos do Desenvolvimento Sustentável, visando à redução do seu uso e o reforço à prevenção e ao tratamento de perturbações do uso de substâncias 20. No Brasil, a meta para o controle de DCNTs e seus fatores de risco visa a uma redução de $10 \%$ na prevalência do consumo abusivo de álcool e de 30\% para o tabagismo 21 . Os dados aqui apresentados evidenciam um cenário brasileiro preocupante, observando-se prevalências de fumo estabilizadas no período recente e de álcool em tendência de crescimento. O aumento no consumo de álcool entre as mulheres, em especial de elevada escolaridade, parece seguir a mesma tendência 
Tabela 2

Índice angular de desigualdade (IAD) para consumo abusivo de álcool e tabagismo de acordo com sexo e região do país. Vigilância de Fatores de Risco e Proteção para Doenças Crônicas por Inquérito Telefônico (Vigitel), Brasil, 2006 a 2017.

\begin{tabular}{|c|c|c|c|c|c|c|c|c|}
\hline \multirow[t]{3}{*}{ Região/Ano } & \multicolumn{4}{|c|}{ Tabagismo } & \multicolumn{4}{|c|}{ Consumo de álcool } \\
\hline & \multicolumn{2}{|c|}{ Homens } & \multicolumn{2}{|c|}{ Mulheres } & \multicolumn{2}{|c|}{ Homens } & \multicolumn{2}{|c|}{ Mulheres } \\
\hline & IAD (\%) & Valor de $p$ & IAD (\%) & Valor de $p$ & IAD (\%) & Valor de $p$ & IAD (\%) & Valor de $p$ \\
\hline Norte & & 0,004 & & 0,074 & & 0,643 & & 0,244 \\
\hline 2006 & $-23,3$ & & $-10,9$ & & 8,8 & & 7,0 & \\
\hline 2007 & $-22,5$ & & $-8,5$ & & 8,6 & & 10,2 & \\
\hline 2008 & $-16,7$ & & $-17,8$ & & 14,2 & & 8,9 & \\
\hline 2009 & $-16,5$ & & $-8,5$ & & 16,2 & & 7,8 & \\
\hline 2010 & $-23,9$ & & $-10,3$ & & 4,2 & & 7,0 & \\
\hline 2011 & $-20,0$ & & $-11,0$ & & 4,5 & & 7,2 & \\
\hline 2012 & $-17,1$ & & $-14,8$ & & 11,3 & & 6,6 & \\
\hline 2013 & $-13,1$ & & $-8,5$ & & 12,0 & & 4,2 & \\
\hline 2014 & $-16,3$ & & $-7,5$ & & 9,7 & & 7,4 & \\
\hline 2015 & $-17,0$ & & $-11,0$ & & 9,6 & & 6,0 & \\
\hline 2016 & $-8,9$ & & $-8,1$ & & 11,0 & & 7,3 & \\
\hline 2017 & $-16,5$ & & $-7,0$ & & 13,7 & & 8,1 & \\
\hline Nordeste & & 0,015 & & 0,002 & & 0,005 & & 0,178 \\
\hline 2006 & $-13,7$ & & $-12,8$ & & 12,8 & & 3,6 & \\
\hline 2007 & $-16,1$ & & $-10,1$ & & 15,4 & & 8,5 & \\
\hline 2008 & $-14,0$ & & $-8,1$ & & 10,7 & & 10,8 & \\
\hline 2009 & $-20,6$ & & $-11,6$ & & 12,1 & & 9,4 & \\
\hline 2010 & $-15,8$ & & $-8,5$ & & 16,5 & & 10,5 & \\
\hline 2011 & $-12,9$ & & $-9,0$ & & 8,8 & & 7,5 & \\
\hline 2012 & $-15,3$ & & $-12,1$ & & 12,5 & & 11,2 & \\
\hline 2013 & $-12,2$ & & $-7,2$ & & 11,2 & & 9,3 & \\
\hline 2014 & $-13,3$ & & $-9,7$ & & 9,4 & & 8,6 & \\
\hline 2015 & $-11,6$ & & $-6,8$ & & 9,9 & & 10,0 & \\
\hline 2016 & $-9,2$ & & $-8,9$ & & 9,5 & & 8,4 & \\
\hline 2017 & $-13,1$ & & $-4,7$ & & 12,1 & & 10,6 & \\
\hline Sudeste & & 0,049 & & 0,162 & & 0,436 & & 0,006 \\
\hline 2006 & $-15,9$ & & $-8,1$ & & 10,3 & & 6,1 & \\
\hline 2007 & $-11,7$ & & $-3,3$ & & 7,7 & & 6,2 & \\
\hline 2008 & $-23,0$ & & $-5,2$ & & 6,9 & & 9,0 & \\
\hline 2009 & $-12,3$ & & $-7,6$ & & 22,0 & & 12,0 & \\
\hline 2010 & $-15,6$ & & $-9,4$ & & 19,4 & & 12,2 & \\
\hline 2011 & $-16,2$ & & $-11,9$ & & 15,8 & & 9,2 & \\
\hline 2012 & $-13,3$ & & $-4,8$ & & 10,6 & & 11,3 & \\
\hline 2013 & $-14,5$ & & $-10,2$ & & 11,7 & & 11,5 & \\
\hline 2014 & $-9,1$ & & $-7,8$ & & 16,7 & & 11,2 & \\
\hline 2015 & $-11,6$ & & $-10,8$ & & 12,4 & & 9,6 & \\
\hline 2016 & $-13,7$ & & $-11,2$ & & 18,8 & & 14,9 & \\
\hline 2017 & $-14,0$ & & $-3,5$ & & 12,1 & & 14,9 & \\
\hline
\end{tabular}

(continua) 
Tabela 2 (continuação)

\begin{tabular}{|c|c|c|c|c|c|c|c|c|}
\hline \multirow[t]{3}{*}{ Região/Ano } & \multicolumn{4}{|c|}{ Tabagismo } & \multicolumn{4}{|c|}{ Consumo de álcool } \\
\hline & \multicolumn{2}{|c|}{ Homens } & \multicolumn{2}{|c|}{ Mulheres } & \multicolumn{2}{|c|}{ Homens } & \multicolumn{2}{|c|}{ Mulheres } \\
\hline & IAD (\%) & Valor de $p$ & IAD (\%) & Valor de $p$ & IAD (\%) & Valor de $p$ & IAD (\%) & Valor de $p$ \\
\hline Sul & & 0,001 & & 0,925 & & 0,069 & & $<0,001$ \\
\hline 2006 & $-31,5$ & & $-6,3$ & & 4,3 & & 8,5 & \\
\hline 2007 & $-16,8$ & & $-7,5$ & & 8,0 & & 10,1 & \\
\hline 2008 & $-20,7$ & & $-8,9$ & & 14,3 & & 7,6 & \\
\hline 2009 & $-18,8$ & & $-9,8$ & & 15,4 & & 11,9 & \\
\hline 2010 & $-19,6$ & & $-10,6$ & & 14,6 & & 13,0 & \\
\hline 2011 & $-21,6$ & & $-15,7$ & & 7,8 & & 9,8 & \\
\hline 2012 & $-15,5$ & & $-15,1$ & & 7 & & 12,2 & \\
\hline 2013 & $-19,0$ & & $-9,7$ & & 17,4 & & 13,1 & \\
\hline 2014 & $-18,2$ & & $-16,6$ & & 14,5 & & 16,4 & \\
\hline 2015 & $-13,2$ & & $-7,4$ & & 16,5 & & 14,6 & \\
\hline 2016 & $-9,7$ & & $-6,0$ & & 19,8 & & 15,2 & \\
\hline 2017 & $-14,1$ & & $-5,1$ & & 12,5 & & 17,4 & \\
\hline Centro-oeste & & $<0,001$ & & 0,013 & & 0,114 & & $<0,001$ \\
\hline 2006 & $-22,8$ & & $-10,7$ & & 5,4 & & 3,2 & \\
\hline 2007 & $-22,6$ & & $-13,9$ & & 8,4 & & 6,9 & \\
\hline 2008 & $-24,5$ & & $-8,3$ & & 4,1 & & 2,6 & \\
\hline 2009 & $-20,4$ & & $-11,7$ & & 13,2 & & 7,7 & \\
\hline 2010 & $-17,5$ & & $-7,6$ & & 14,3 & & 10,6 & \\
\hline 2011 & $-20,2$ & & $-8,8$ & & 11,9 & & 9,6 & \\
\hline 2012 & $-16,6$ & & $-9,1$ & & 14,5 & & 7,6 & \\
\hline 2013 & $-14,6$ & & $-8,3$ & & 11,7 & & 7,6 & \\
\hline 2014 & $-21,6$ & & $-9,2$ & & 2,4 & & 10,7 & \\
\hline 2015 & $-15,1$ & & $-9,7$ & & 13,2 & & 21,8 & \\
\hline 2016 & $-18,7$ & & $-7,9$ & & 20,2 & & 17,2 & \\
\hline 2017 & $-6,8$ & & $-9,3$ & & 12,6 & & 17,1 & \\
\hline
\end{tabular}

Nota: valor de p obtido com base na regressão de Prias-Winsten.

do consumo do tabaco no passado, quando as mulheres iniciaram o hábito de fumar em um processo de afirmação em busca de emancipação, independência e aproximação ao universo masculino 22,23 . Nesse sentido, o aumento do apoio às estratégias focadas no controle desses fatores deve ser priorizado. Munhoz et al. 9 , em um artigo avaliando as tendências na prevalência do consumo abusivo de álcool até 2013 também utilizando dados do Vigitel, não haviam encontrado evidências de aumento na prevalência para mulheres. Verificando nossos dados detalhadamente (análises não apresentadas), observamos que o crescimento no consumo de álcool ocorreu apenas após a inserção dos anos 2016 e 2017 na análise, evidenciando assim que este pode ser um comportamento bem mais comum nos últimos anos.

Quanto às desigualdades, este estudo identificou um maior consumo abusivo de álcool entre os mais escolarizados. Grittner et al. 24, em um estudo com dados de 33 países, encontraram odds ratio (OR) de consumo de álcool no último ano maior para os indivíduos com maior escolaridade comparados aos menos escolarizados [OR para homens $=1,50(1,31 ; 1,73)$; OR para mulheres = 1,99 $(1,76$; 2,24)]. Por outro lado, para o consumo abusivo de álcool não foi encontrada significância estatística na medida combinada de todos os países analisados. Os autores mostraram que a variabilidade desse desfecho segundo a escolaridade nos países foi alta, ora o consumo sendo maior entre os mais ricos, ora entre os mais pobres. Ainda, observando somente os países de renda baixa, o estudo encontrou um maior consumo abusivo entre as mulheres mais escolarizadas e os homens menos escolarizados 24. Globalmente, esse tipo de consumo tem sido descrito pela OMS como tendo determinantes 
diversos, incluindo fatores de risco individuais, como sexo e idade, por exemplo, e ambientais, como disponibilidade de álcool, ambiente político, existência de legislação específica e situação econômica de um país 18. Um estudo com dados da Pesquisa Nacional de Saúde (PNS) de 2013, que inclui amostra representativa do país, encontrou uma prevalência de consumo abusivo de álcool 1,2 vez maior para os homens com nível superior comparados aos sem instrução 25. Para as mulheres, a prevalência do fator de risco foi 2,3 vezes maior para aquelas mais escolarizadas, quando comparadas às menos escolarizadas 25 . Outro estudo, que incluiu uma amostra de capitais brasileiras, mas com adolescentes, também encontrou uma prevalência de consumo abusivo de álcool 2,8 vezes maior entre os mais ricos 26 . Destaca-se também a limitação desse indicador, considerando que mede o álcool consumido em uma única ocasião de forma abusiva, comum em populações jovens e de maior escolaridade, em momentos de celebração, não necessariamente referindo a um consumo crônico, ou de dependência, o que é geralmente mais comum entre os mais pobres 19.

Ainda cabe destacar alguns pontos sobre a variável de álcool analisada no presente estudo. O consumo abusivo de álcool é diferente do consumo crônico ou alcoolismo. Consumo abusivo em uma única ocasião, embora possa ser um comportamento relativamente frequente em grupos de jovens com maior escolaridade, comum em celebrações, está associado a uma série de problemas de saúde. Uma revisão da literatura sobre o assunto identificou que o consumo abusivo de álcool em uma única ocasião aumentou o risco de doenças cardiovasculares e metabólicas, pior saúde mental, doenças neurológicas, além de consequências agudas como morte por intoxicação etílica, lesão física, acidentes de trânsito, brigas, comportamento violento e sexo sem proteção 27,28. Dessa forma, embora seja diferente do consumo crônico de álcool, ele ainda representa um risco importante à saúde, principalmente por afetar um grupo social diferente do afetado por outros comportamentos de risco. Portanto, políticas que reduzam o consumo crônico e o abusivo em uma única ocasião parecem ter grupos focais diferentes, exigindo estratégias e planejamento diferenciados.

Atualmente, o Brasil é classificado pelo Banco Mundial como um país de renda média-alta e passa por diversas mudanças sociais. Os resultados do presente estudo mostram que as desigualdades para o consumo abusivo de álcool em mulheres seguem o padrão dos países de baixa renda 24 , mas para os homens os resultados não são tão claros, considerando que os mais escolarizados ainda apresentavam maior consumo em 2017 quando comparamos às diferenças encontradas em 2006. Tanto a produção quanto a venda de bebidas alcoólicas do Brasil têm aumentado e a projeção do consumo até 2020 também prevê aumento 29 . O aumento do consumo pode justificar também o aumento da desigualdade, visto que indivíduos com maior escolaridade em geral apresentam também maior poder aquisitivo e por sua vez exercem maior poder de compra quando comparados aos menos escolarizados. Entre os homens, isso não é fortemente evidente porque provavelmente o consumo foi sempre alto, mas nas mulheres em que o consumo claramente mostrou um importante aumento, essa poderia ser uma das possíveis explicações para o crescimento também das desigualdades, considerando que o consumo entre os menos escolarizados se manteve estável. Além disso, é possível que o aumento do consumo tenha ocorrido para um grupo específico das mulheres como as mais jovens.

A respeito do tabagismo, as estimativas mostram que a prevalência vem diminuindo ao longo dos anos para ambos os sexos. Em grande parte, isso se deve às políticas de controle do tabagismo no país, como por exemplo, a implementação de imagens de advertência, restrição de publicidade, aumento de impostos para o cigarro, entre outras 30,31. O controle de tabaco no Brasil tem sido efetivo ao longo dos anos, porém, mesmo com a prevalência total diminuindo, as desigualdades ainda persistem. O grupo menos escolarizado ainda é o que apresenta os maiores porcentuais de tabagismo com um padrão muito bem definido de bottom inequality, quando um grupo menos favorecido apresenta indicadores muito piores do que os grupos intermediários e do extremo oposto. Um estudo sobre desigualdades do tabagismo no Brasil com amostra de adolescentes revela que as diferenças socioeconômicas na prevalência de fumo são marcadas desde idades precoces e estão relacionadas a taxas de abandono escolar, repetência e entrada precoce no mercado de trabalho. Isso poderia explicar o padrão dos menos escolarizados apresentando prevalências superiores de tabagismo quando adultos 32. É importante ressaltar que as políticas de controle do tabaco devem atingir com maior efetividade a parcela da população mais afetada, a fim de reduzir também as desigualdades deste comportamento de risco. 
O Brasil é um país com grande multiplicidade cultural e isto se reflete também nos dados de saúde. O aumento nas desigualdades do consumo abusivo de álcool ocorreu no Sul para homens e em quase todas as regiões brasileiras para as mulheres, exceto na Região Norte. Para o tabagismo, os resultados sugeriram uma diminuição na desigualdade para homens em todas regiões exceto na Sudeste e na Centro-oeste. Já para as mulheres, redução apenas na Região Norte. A prevalência de consumo abusivo de álcool para as mulheres da Região Norte foi a única que apresentou uma diminuição ao longo do tempo (dados não apresentados). Esse resultado pode sugerir que enquanto no resto do país houve um aumento do consumo abusivo de álcool para mulheres, principalmente nos grupos mais ricos, no Norte isto não foi evidente. Um estudo sobre desigualdades no tabagismo com dados da Pesquisa Nacional por Amostra de Domicílios (PNAD) de 2008 identificou importantes diferenças em termos de prevalência, com a Região Sul apresentando as maiores frequências e a Norte as menores 11. O mesmo estudo observou maior prevalência de fumo no quintil mais pobre para todas as regiões, no mesmo sentido dos resultados encontrados neste trabalho ${ }^{11}$. Com os achados do presente estudo ainda é difícil afirmar se as desigualdades têm um padrão diferente para algumas regiões do país ou não, porém, é importante manter o monitoramento a fim de identificar possíveis localidades que precisem de intervenções direcionadas.

Este trabalho apresenta potencialidades no sentido da representatividade das capitais brasileiras durante um longo período de tempo, o que permite o monitoramento dos desfechos estudados e suas desigualdades, auxiliando no planejamento de políticas e estratégias de intervenção. Diferenças no consumo de álcool entre os estratos de escolaridade já foram evidenciadas na literatura anteriormente, mas apenas para o período de 2006 a 2013 9. Além da extensão do período de análise no presente estudo, cabe salientar que utilizamos testes formais para avaliação das medidas de desigualdade. Entretanto, algumas limitações precisam ser mencionadas. A primeira delas é relativa à utilização da escolaridade como medida proxy da posição socioeconômica. O Vigitel não coleta informações sobre bens ou renda, o que impossibilita a avaliação das desigualdades socioeconômicas com uma medida mais efetiva. Embora a escolaridade não seja uma medida direta de posição socioeconômica, ela pode refletir o conhecimento em relação a hábitos saudáveis, maior acesso a serviços de saúde e maior acúmulo de capital humano, aspectos estes fortemente relacionados a classes privilegiadas 33,34 . Ainda assim, estudos que possam avaliar a tendência com outras dimensões de desigualdade ainda são uma lacuna na literatura a ser explorada. Além disso, como este trabalho é baseado em entrevistas por telefone fixo, é possível que justamente a parcela mais pobre da população não esteja incluída no estudo, o que resulta em uma subestimação das desigualdades aqui apresentadas, apesar de o ajuste para pesos amostrais minimizar este viés. Ainda, o perfil sociodemográfico da população que vive em domicílios que têm somente telefones celulares difere daquele das pessoas com linha telefônica fixa, apresentando maior frequência de indivíduos mais jovens e com escolaridade até o Ensino Médio. Em contrapartida, a proporção de homens e mulheres que vivem em domicílios com apenas telefone celular ou com telefone fixo é semelhante. Para as regiões geográficas, foi observado que a pesquisa Vigitel subestimou a prevalência de fumantes no Norte e no Nordeste em 2013, por exemplo, em comparação à PNS 35. Mesmo assim, é importante observar que mesmo com essas limitações foram identificadas diferenças relevantes entre os mais e menos escolarizados, e este padrão pode ser muito mais extremo excluindo estes problemas metodológicos.

Frente ao cenário apresentado neste estudo, cabe destacar que embora o plano nacional de enfrentamento às DCNTs inclua a redução do tabagismo e do consumo de álcool por meio de estratégias como o aumento dos preços e impostos sobre os produtos, entre outros aspectos 21 , nenhum avanço neste sentido parece estar ocorrendo nos últimos anos, com o tabagismo estagnado e com o crescimento do consumo de álcool entre as mulheres. Ainda, a previsão para a redução das DCNTs até 2030 não é promissora e provavelmente não será alcançada ${ }^{36}$. Em relação a políticas específicas para drogas lícitas e ilícitas, existe, desde 2006, o Sistema Nacional de Políticas Públicas sobre Drogas e, em abril de 2019, foi instaurada a nova política para estas drogas, voltada à população brasileira como um todo. Apesar de não estar claro quais indicadores sociais são explorados, ou ainda se diferenças regionais são consideradas, a nova política indica que sua execução leva em conta a priorização de comunidades mais vulneráveis 37 . Nesse sentido, é possível que, até o momento do presente estudo, tenha havido menor preocupação com a população que mais consome álcool, por exemplo os mais escolarizados. 
A partir deste momento e nos próximos anos, é imprescindível que o monitoramento de fatores de risco, como o consumo de álcool e o tabagismo, bem como de suas desigualdades, seja continuado. O SUS, um dos principais responsáveis no país pelo combate às DCNTs e seus fatores de risco, é também um dos mais afetados por políticas atuais de congelamento de gastos com a saúde 38,39. Além disso, medidas regulatórias de controle de fatores de risco, recomendadas pela OMS, como o aumento de preços e de impostos de produtos nocivos à saúde não têm sido implementadas 40. Desde 2017, cigarros não tiveram ajuste dos preços e a Portaria no 263, de 23 de março de 2019 41, no âmbito do Ministério da Justiça e Segurança Pública, instituiu um Grupo de Trabalho para avaliar tanto a conveniência como a oportunidade da redução da tributação de cigarros fabricados no Brasil. Estimativas mostram que um aumento de $10 \%$ em impostos nos preços do tabaco poderia diminuir o consumo de tabaco em até $8 \% 42$.

O consumo abusivo de álcool e o tabagismo mostram padrões inversos de desigualdade socioeconômica usando-se a escolaridade como proxy, com o primeiro atingindo mais os indivíduos com maior nível socioeconômico e o segundo, aqueles com menor nível socioeconômico. Além disso, o consumo abusivo de álcool vem crescendo ao longo dos anos e o tabagismo diminuindo. Embora avanços tenham sido feitos na área do tabagismo, as desigualdades socioeconômicas persistem e estão presentes também em relação ao consumo de álcool. Tendo em vista que as populações mais afetadas pelos dois desfechos são opostas, estratégias específicas para cada um dos problemas deveriam levar em conta este achado, a fim de reduzir as desigualdades existentes.

\section{Colaboradores}

A. Wendt, C. S. Costa, F. S. Costa, D. C. Malta e I. Crochemore-Silva participaram da análise, interpretação dos dados e redação do texto, revisaram criticamente o manuscrito, aprovaram a versão final e concordou com todos os aspectos relacionados à integridade do trabalho.

\section{Informações adicionais}

ORCID: Andrea Wendt (0000-0002-4640-2254); Caroline S. Costa (0000-0003-0281-6890); Francine S. Costa (0000-0001-9558-937X); Deborah Carvalho Malta (0000-0002-8214-5734); Inácio Crochemore-Silva (0000-0001-5390-8360).

\section{Referências}

1. Malta DC, Andrade SSCA, Oliveira TP, Moura L, Prado RR, Souza MFM. Probabilidade de morte prematura por doenças crônicas não transmissíveis, Brasil e regiões, projeções para 2025. Rev Bras Epidemiol 2019; 22:e190030.

2. Castro MC, Massuda A, Almeida G, MenezesFilho NA, Andrade MV, Noronha KVMS, et al. Brazil's unified health system: the first 30 years and prospects for the future. Lancet 2019; 394:345-56.

3. Viacava F, Porto SM, Carvalho CC, Bellido JG. Desigualdades regionais e sociais em saúde segundo inquéritos domiciliares (Brasil, 19982013). Ciênc Saúde Colet 2019; 24:2745-60.

4. Organização Pan-Americana da Saúde. 10 principais causas de morte no mundo. https:// www.paho.org/bra/index.php?option $=\mathrm{com}_{-}$ content\&view $=$ article \&id $=5638: 10$-princi pais-causas-de-morte-no-mundo\&Itemid $=0$ (acessado em 27/Jul/2019).

5. Di Cesare M, Khang YH, Asaria P, Blakely T, Cowan MJ, Farzadfar F, et al. Inequalities in non-communicable diseases and effective responses. Lancet 2013; 381:585-97.

6. Stringhini S, Sabia S, Shipley M, Brunner E, Nabi H, Kivimaki M, et al. Association of socioeconomic position with health behaviors and mortality. JAMA 2010; 303:1159-66. 
7. Ministério da Saúde. A política do Ministério da Saúde para atenção integral a usuários de álcool e outras drogas. Brasília: Ministério da Saúde; 2003.

8. Malta DC, Iser BPM, Sá NNB, Yokota RTC, Moura L, Claro RM, et al. Tendências temporais no consumo de tabaco nas capitais brasileiras, segundo dados do VIGITEL, 2006 a 2011. Cad Saúde Pública 2013; 29:812-22.

9. Munhoz TN, Santos IS, Nunes BP, Mola CL, Silva ICM, Matijasevich A. Tendências de consumo abusivo de álcool nas capitais brasileiras entre os anos de 2006 a 2013: análise das informações do Vigitel. Cad Saúde Pública 2017; 33:e00104516.

10. Departamento de Vigilância de Doenças e Agravos não Transmissíveis e Promoção da Saúde, Secretaria de Vigilância em Saúde, Ministério da Saúde. Vigitel Brasil 2019. Vigilância de fatores de risco e proteção para doenças crônicas por inquérito telefônico. Estimativas sobre frequência e distribuição sociodemográfica de fatores de risco e proteção para doenças crônicas nas capitais dos 26 estados brasileiros e no Distrito Federal em 2019. Brasília: Ministério da Saúde; 2020.

11. Barros AJD, Cascaes AM, Wehrmeister FC, Martínez-Mesa J, Menezes AMB. Tabagismo no Brasil: desigualdades regionais e prevalência segundo características ocupacionais. Ciênc Saúde Colet 2011; 16:3707-16.

12. Departamento de Vigilância de Doenças e Agravos não Transmissíveis e Promoção da Saúde, Secretaria de Vigilância em Saúde, Ministério da Saúde. Vigitel. Brasil 2017. Vigilância de fatores de risco e proteção para doenças crônicas por inquérito telefônico. Estimativas sobre frequência e distribuição sociodemográfica de fatores de risco e proteção para doenças crônicas nas capitais dos 26 estados brasileiros e no Distrito Federal em 2017. Brasília: Ministério da Saúde; 2017.

13. Ministéro da Saúde. Ponderação pelo método rake.http://svs.aids.gov.br/bases_vigitel_viva/ dic_var/vigitel/Ponderacao_Metodo-Rake.pdf (acessado em 11/Jul/2020).

14. Antunes JLF, Cardoso MRA. Uso da análise de séries temporais em estudos epidemiológicos. Epidemiol Serv Saúde 2015; 24:565-76.

15. Silva ICM, Restrepo-Mendez MC, Costa JC, Ewerling F, Hellwig F, Ferreira LZ, et al. Mensuração de desigualdades sociais em saúde: conceitos e abordagens metodológicas no contexto brasileiro. Epidemiol Serv Saúde 2018; 27:1-12.

16. World Health Organization. Handbook on health inequality monitoring with a special focus on low-and middle-income countries. https://apps.who.int/iris/han dle/10665/85345 (acessado em 11/Fev/2021).

17. Barros AJ, Victora CG. Measuring coverage in $\mathrm{MNCH}$ : determining and interpreting inequalities in coverage of maternal, newborn, and child health interventions. PLoS Med 2013; 10:e1001390.
18. Alcohol use and burden for 195 countries and territories, 1990-2016: a systematic analysis for the Global Burden of Disease Study 2016. Lancet 2018; 392:1015-35.

19. World Health Organization. Global status report on alcohol and health. Geneva: World Health Organization; 2018.

20. United Nations. Sustainable development goals. https://sustainabledevelopment.un.org/ sdgs (acessado em 29/Ago/2019).

21. Malta DC, Silva Jr. JB. O Plano de Ações Estratégicas para o Enfrentamento das Doenças Crônicas Não Transmissíveis no Brasil e a definição das metas globais para o enfrentamento dessas doenças até 2025: uma revisão. Epidemiol Serv Saúde 2013; 22:151-64.

22. Apelberg B AM, Asma S, Donaldson E, Yeong CC, Vaithinathan R. Prevalence of tobacco use and factors influencing initiation and maintenance among women. In: Samet JM, Yoon S-Y, editors. Gender, women, and the tobacco epidemic. Geneva: World Health Organization; 2010. p. 29-50.

23. Malta DC, Stopa SR, Santos MAS, Andrade SSCA, Oliveira TP, Cristo EB, et al. Evolução de indicadores do tabagismo segundo inquéritos de telefone, 2006-2014. Cad Saúde Pública 2017; 33 Suppl 3:e00134915.

24. Grittner U, Kuntsche S, Gmel G, Bloomfield $\mathrm{K}$. Alcohol consumption and social inequality at the individual and country levels - results from an international study. Eur J Public Health 2013; 23:332-39.

25. Garcia LP, Freitas LRS. Consumo abusivo de álcool no Brasil: resultados da Pesquisa $\mathrm{Na}$ cional de Saúde 2013. Epidemiol Serv Saúde 2015; 24:227-37.

26. Sanchez ZM, Locatelli DP, Noto AR, Martins SS. Binge drinking among Brazilian students: a gradient of association with socioeconomic status in five geo-economic regions. Drug Alcohol Depend 2013; 127:87-93.

27. Adan A, Benaiges I, Forero DA. Heavy episodic drinking or binge drinking: a booming consumption pattern. In: Preedy VR, editor. Neuropathology of drug addictions and substance misuse. San Diego: Academic Press; 2016; p. 389-97.

28. Mendonça AKRH, Jesus CVF, Figueiredo MBGA, Valido DP, Nunes MAP, Lima SO. Consumo de álcool e fatores associados ao binge drinking entre universitárias da área de saúde. Esc Anna Nery Rev Enferm 2018; 22:e20170096.

29. Viana FLE. Indústria de bebidas alcoólicas. Caderno Setorial ETENE 2017; 2:2-14.

30. Levy D, Almeida LM, Szklo A. The Brazil SimSmoke policy simulation model: the effect of strong tobacco control policies on smoking prevalence and smoking-attributable deaths in a middle income nation. PLoS Med 2012; 9:e1001336. 
31. Silva LC, Araujo AJ, Queiroz AM, Sales MD, Castellano MV. Smoking control: challenges and achievements. J Bras Pneumol 2016; 42:290-8.

32. Barreto SM, de Figueiredo RC, Giatti L. Socioeconomic inequalities in youth smoking in Brazil. BMJ Open 2013; 3:e003538.

33. Liberatos P, Link BG, Kelsey JL. The measurement of social class in epidemiology. Epidemiologic Rev 1988; 10:87-121.

34. Galobardes B, Shaw M, Lawlor DA, Lynch JW, Davey SG. Indicators of socioeconomic position (part 1). J Epidemiol Community Health 2006; 60:7-12.

35. Bernal RTI, Malta DC, Claro RM, Monteiro CA. Effect of the inclusion of mobile phone interviews to Vigitel. Rev Saúde Pública 2017; 51 Suppl 1:15s.

36. Malta DC, Silva AG, Teixeira RA, Machado IE, Coelho MRS, Hartz ZMA. Avaliação do alcance das metas do plano de enfrentamento das doenças crônicas não transmissíveis no Brasil, 2011-2022. Anais do Instituto de Higiene e Medicina Tropical 2019; Suplemento 1:S9-S16.

37. Brasil. Decreto no 9.761, de 11 de abril de 2019. Aprova a Política Nacional sobre Drogas. Diário Oficial da União 2019; 11 abr.

38. Malta DC, Duncan BB, Barros MBA, Katikireddi SV, Souza FM, Silva AG, et al. Fiscal austerity measures hamper noncommunicable disease control goals in Brazil. Ciênc Saúde Colet 2018; 23:3115-22.
39. Brasil. Emenda Constitucional no 95, de 15 de dezembro de 2016. Altera o Ato das Disposições Constitucionais Transitórias, para instituir o Novo Regime Fiscal, e dá outras providências. Diário Oficial da União 2016; 15 dez.

40. World Health Organization. Tackling NCDs: 'best buys' and other recommended interventions' for the prevention and control of noncommunicable diseases. Geneva: World Health Organization; 2017.

41. Ministério da Justiça e Segurança Pública. Portaria no 263, de 23 de março de 2019. Institui Grupo de Trabalho, no âmbito do Ministério da Justiça e Segurança Pública, para avaliar a conveniência e oportunidade da redução da tributação de cigarros fabricados no Brasil. Diário Oficial Brasil 2019; 26 mar

42. Organização Mundial da Saúde; Organização Pan-Americana da Saúde. MPOWER: um plano de medidas para reverter a epidemia de tabagismo. https://www.paho.org/bra/index. php?option $=$ com_docman\&view $=$ downloa d\&alias $=375$ - power-um-plano-para-rever ter-a-epidemia-tabagismo-5\&category_slug= tabagismo-132\&Itemid $=965$ (acessado em 20/ Mai/2019). 


\section{Abstract}

This was a time trend study aimed at assessing inequalities in smoking and abusive alcohol consumption, considering schooling as a proxy for socioeconomic status, according to sex and region of Brazil. The study used data from the Risk and Protective Factors Surveillance System for Chronic Non-Comunicable Diseases Through Telephone Interview (Vigitel) survey collected from 2006 to 2017. The outcomes were abusive alcohol consumption and smoking. The inequalities were assessed based on schooling, with double stratification by sex and major geographic region. Weighted linear least squares regression was used to assess the trend in prevalence of outcomes and their inequalities. Prevalence of abusive alcohol consumption increased in women, from $7.8 \%$ in 2006 to $12.2 \%$ in 2017 and was consistently higher among individuals with more schooling. Prevalence of smoking decreased in both men and women and was consistently higher among those with less schooling. Inequality in alcohol consumption between schooling groups appears to have increased over time, higher in women, but inequality in smoking appears to have decreased. In some regions of Brazil, the measures of inequality suggest stability and in others an increase in inequality in alcohol consumption (Southeast, South, and Central regions in women) and a decrease in smoking (all regions except the Southeast in men; Northeast and Central in women). Despite the strides in smoking reduction, inequalities persist in schooling and are also present in alcohol consumption. The outcomes show opposite trends, so strategies should focus on each of the problems in order to reduce existing inequalities.

Tobacco Use Disorder; Risk Factors; Chronic Disease; Health Inequality Indicators

\section{Resumen}

Estudio de tendencia temporal, con el objetivo de evaluar las desigualdades en el tabaquismo y consumo abusivo de alcohol, considerando la escolaridad como proxy de nivel socioeconómico, de acuerdo con el sexo y regiones brasileñas. Se utilizaron datos de Vigilancia de Factores de Riesgo y Protección para Enfermedades Crónicas No Transmisibles por Entrevista Telefónica (Vigitel), recogidos entre 2006 y 2017. Los resultados fueron el consumo abusivo de bebidas alcohólicas y tabaco. Las desigualdades se evaluaron a partir de la escolaridad, con doble estratificación por sexo y regiones brasileñas. Para evaluar la tendencia de las prevalencias de los resultados y sus desigualdades se utilizó la regresión lineal por mínimos cuadrados ponderados de la variancia. La prevalencia de consumo abusivo de alcohol aumentó en mujeres de 7,8\% (2006) a 12,2\% (2017) y fue siempre mayor entre los más escolarizados. La prevalencia de tabaquismo disminuyó en hombres y mujeres, $y$ siempre fue mayor entre los menos escolarizados. La desigualdad en el consumo de alcohol entre los grupos de escolaridad parece que ha aumentado a lo largo del tiempo, con mayor magnitud entre las mujeres, pero la desigualdad en el tabaquismo parece estar disminuyendo. En algunas regiones del país, las medidas de desigualdad sugieren estabilidad y en otras un aumento de la desigualdad en el consumo de alcohol (Sudeste, Sur y Centro-oeste, entre las mujeres) y disminución en el tabaquismo (todas las regiones, excepto la Sudeste, entre los hombres; Nordeste y Centro-oeste, entre las mujeres). A pesar de los avances en la reducción del tabaquismo, las desigualdades en la escolaridad persisten y están presentes también en relación con el alcohol. Los resultados tienen comportamientos opuestos, por lo tanto, las estrategias deben centrarse en cada uno de los problemas, a fin de reducir las desigualdades existentes.

Tabaquismo; Factores de Riesgo; Enfermedad Crónica; Indicadores de Desigualdad en Salud
Recebido em 18/Mar/2020

Versão final reapresentada em 11/Jul/2020

Aprovado em 28/Jul/2020 